\title{
Small Brane Black Holes in Randall-Sundrum type I Scenario
}

\author{
D. Karasik* C. Sahabandu, P. Suranyi, and L. C. R. Wijewardhana \\ Department of Physics, University of Cincinnati, Cincinnati, Ohio
}

\begin{abstract}
An approximation method to study the properties of a small black hole located on the TeV brane in the Randall-Sundrum type I scenario is presented. The method enables us to find the form of the metric close to the matter distribution when its asymptotic form is given. The short range solution is found as an expansion in the ratio between the Schwarzschild radius of the black hole and the curvature length of the bulk. Long range properties are introduced using the linearized gravity solution as an asymptotic boundary condition. The solution is found up to first order. It is valid in the region close to the horizon but is not valid on the horizon. The regularity of the horizon is still under study.
\end{abstract}

\section{INTRODUCTION}

Within the Randall-Sundrum brane world scenarios [1, 2] the four dimensional universe is viewed as a three-brane with brane tension $\sigma$, embedded in a AdS five-dimensional bulk, with a cosmological constant $\Lambda$. A flat brane is achieved by fine-tuning the brane tension and the cosmological constant

$$
8 \pi G_{5} \Lambda=-\frac{6}{\ell^{2}} ; 8 \pi G_{5} \sigma= \pm \frac{6}{\ell},
$$

where $\ell$ is the curvature length of the AdS bulk.

The Randall-Sundrum I (RSI) scenario was postulated [1] to solve the hierarchy problem by making gravity strong at the weak scale (of a $\mathrm{TeV}$ ). In this scenario the world consists of two opposite tension branes embedded in an ADS bulk with an orbifold structure. The negative tension brane is called the TeV brane, while the positive tension brane is called the Planck brane. $Z_{2}$ orbifold symmetry is assumed about each brane. The ratio between the TeV electro-weak scale and the Planck scale is taken to be $\lambda \sim 10^{-16}$. In this scenario the standard model particles are assumed to propagate only on the $\mathrm{TeV}$ brane. Gravity, on the other hand, is five dimensional and propagates in the bulk. The five dimensional gravitational coupling is of order $1 \mathrm{TeV}, G_{5}=\left(M_{5}\right)^{-3}, M_{5} \sim 1 \mathrm{TeV}$. The hierarchy appears on the $\mathrm{TeV}$ brane at distances much larger than the curvature length $\ell$. The gravitational attraction becomes ordinary four-dimensional attraction with Newton's constant $G_{4}=\left(M_{\mathrm{Pl}}\right)^{-2}, M_{\mathrm{Pl}}=10^{16} \mathrm{TeV}$.

RSII consists of a single positive tension brane with $Z_{2}$ symmetry about the brane. This model does not yield a low scale gravitational theory. From now on we restrict our attention to the RSI scenario.

Black holes in theories with extra dimensions have been studied widely. Myers and Perry [3] found Schwarzschild type solutions (MPS) in $D$-dimensional asymptotically flat space. Black hole solutions were also found in asymptotically AdS space [4, [5]. No non-trivial closed form black hole solutions, other than the black string solution [6], which extends in a uniform manner from the brane into the extra dimension, have been found in three-brane theories of the Randall-Sundrum type. Given that there is considerable interest surrounding the production of black holes at accelerators [7], and in collisions of cosmic rays [8], it is important to develop approximate methods to find black hole solutions in Randall-Sundrum brane world theories.

Some initial attempts at finding black hole solutions centered on deriving the induced metric on the brane by solving the Hamiltonian constraint conditions [9]. Some of the induced solutions do not arise from matter distributions confined to the brane [10]. Linearized solutions about RS backgrounds [1] as well as numerical solutions [12] have also been derived. In a recent paper, Casadio and Mazzacurati [13] investigated how solutions of the Einstein equation in RSII models propagate into the bulk by using a asymptotic expansion of metric coefficients in $r^{-1}$, where $r$ is the radial distance on the brane. They reduced the Einstein equations to a set of differential equations involving the expansion coefficients which are functions of the bulk coordinate $w$. Solving these equations they found a family of solutions parameterized by two physical parameters, the mass $M$ and the post-Newtonian parameter $\eta$.

It would be interesting and important to find black hole solutions in RSI models with TeV scale gravity. We are especially interested in how such solutions evolve when the black hole mass increases starting from around a $\mathrm{TeV}$ (where the flat space MPS limit is valid) to macroscopic scales. From the point of view of cosmology it is useful to

*Electronic address: karasik@physics.uc.edu 
understand how $\mathrm{TeV}$ scale primordial black holes accrete or radiate matter when immersed in a hot plasma. For that we need to work out their thermodynamic properties. That requires a detailed determination of the area of the event horizon as a function of mass. As the size of the black hole approaches the AdS radius, which is of the same order as the length of separation of the two branes [8], the character of the solution should change and Gregory-Laflamme type instabilities [14] could materialize. One has to find bulk profiles of TeV mass black holes to address these issues.

A TeV black hole solution in RSI scenario is characterized by two scales: The curvature length of the bulk $\ell$, generated by the bulk cosmological constant and the five dimensional Schwarzschild radius, $\mu$, related to the mass of the black hole as $\mu=\sqrt{8 G_{5} M /(3 \pi)}$. For a reasonable setup [15] the curvature length in RSI is of order $\ell \sim 10(\mathrm{TeV})^{-1}$, while the "mass" can range from a TeV black hole $\mu \sim 1(\mathrm{TeV})^{-1}$, to ordinary black holes $\mu \sim 10^{16}(\mathrm{TeV})^{-1}$. Clearly, one should use an appropriate approximation method for various values of the parameters $\ell, \mu$.

Linearized gravity is valid when the scales under study are much larger than the Schwarzschild radius $r \gg \mu$. Einstein equations are expanded to first order in $\mu^{2} \sim G_{5} M$, and the solution appears to be a superposition of various modes; the zero mode, the massive modes, and the radion. The radion appears to be a four dimensional massless mode which describes the relative motion of the branes. Being massless, the radion mediates a long range strong gravitational force and therefore ruins the hierarchy. Provided a stabilization mechanism which in general makes the radion massive, the linearized solution demonstrates the transition between five dimensional TeV scaled gravity, at distances smaller than the curvature length, and four dimensional Planck scale gravity at distances larger than the curvature length along the $\mathrm{TeV}$ brane. The advantage of the asymptotically conformally flat linearized solution is to enable the definition of conserved momenta and identification of mass (in analog to the ADM mass). On the other hand, one is unable to study the horizon of the black hole using linearized gravity, since the horizon is located approximately at $r \sim \mu$. Linearized gravity in RSI brane scenario was widely studied [1, 2, 16, 17, 18] and will be presented in appendix A] Asymptotic expansion in $r^{-1}[13]$ is valid only when $r \ll \ell, \mu$. In cases where $\mu<\ell$ the expansion can not be used for $r<\ell$, and will not enable us to study the horizon.

In this paper we use the following method: we expand the solution in the dimensionless parameter $\epsilon=\mu / \ell \ll 1$. The coordinates are rescaled by $\mu$, therefore, an expansion in $\epsilon$ actually means an expansion in inverse powers of $\ell$. Zeroth order in $\epsilon$ should be nothing but the five dimensional flat background MPS black hole [3]. First order in $\epsilon$ "turns on" the brane tension, while second order in $\epsilon$ "turns on" the bulk cosmological constant.

Up to first order in $\epsilon$ ( $\epsilon$ solution) the metric is found in section [II The $\epsilon$ solution is required to be regular everywhere. Especially it should not include black strings off the brane. Naturally, the $\epsilon$ solution is valid in the region $r \ll \ell$. It includes the region close to the horizon which is essential for horizon studies. However, it is not valid in the asymptotic region $r \rightarrow \infty$ and one is unable to identify the mass or satisfy the junction conditions on the second brane. In order to overcome these subtleties we use the linearized solution as an asymptotic boundary condition. Linearized gravity is valid in the region $r \gg \mu$. We are interested in the case $\mu \ll \ell$, therefore, there is an intermediate region $\mu \ll r \ll \ell$ where the $\epsilon$ solution and the linearized solution coincide. In this intermediate range we take the large distance behavior of the $\epsilon$ solution and compare it to the short distance behavior of the linearized solution. As a result, the long range characteristics (mass identification and second brane conditions) are introduced into our short ranged $\epsilon$ solution.

In section III we analyze the properties of the horizon. Clearly, the MPS solution, (zero order in $\epsilon$ ), includes a regular horizon with a well defined temperature. The horizon is modified in the $\epsilon$ solution. Although the $\epsilon$ solution is valid in the region close to the horizon, it is not obvious that the new horizon is regular. The $\epsilon$ solution acquires a null surface defined by $g_{t t}=0$. The normal vector is null and the static Killing vector is null, i.e. the surface is a Killing Horizon. The surface gravity of the horizon is not a constant which indicates that the horizon is singular [19]. This leaves us with the following possibilities; (1) The horizon is singular. Which means that there are no small black holes in Randall-Sundrum scenario. (2) The $\epsilon$ expansion does not converge on the horizon. It is valid only at a distance $\epsilon$ away from the horizon, and therefore one cannot use it to calculate the surface gravity. (3) The boundary conditions should be changed thus changing the entire solution. The issue of the regularity of the horizon is under study and the detailed discussion is left for a future publication.

\section{AN EXPANSION CLOSE TO THE HORIZON}

The goal of this paper is to find a method to explore the horizon of a small black hole in RSI scenario. The configuration is characterized by two length scales; the five dimensional Schwarzschild radius $\mu\left(\sim 1 \mathrm{TeV}^{-1}\right)$, and the AdS curvature length $\ell\left(\sim 10 \mathrm{TeV}^{-1}\right)[15]$. The only dimensionless parameter is the ratio between the two $\epsilon=\mu / \ell$. For a small black hole $\epsilon$ is smaller than 1 and the metric can be expanded in $\epsilon$.

The zero order metric is just the MPS solution

$$
d \bar{s}^{2}=-\left(1-\frac{\mu^{2}}{\bar{\rho}^{2}}\right) d \bar{t}^{2}+\left(\frac{\bar{\rho}^{2}}{\bar{\rho}^{2}-\mu^{2}}\right) d \bar{\rho}^{2}+\bar{\rho}^{2} d \psi^{2}+\bar{\rho}^{2} \sin ^{2} \psi d \Omega_{2}^{2} .
$$


Where we use spherical coordinates in the $(4+1)$-dimensional bulk $(\bar{t}, \bar{\rho}, \psi, \theta, \phi)$. For convenience we rescale the radial coordinate $\bar{\rho}$ and the time coordinate $\bar{t}$ by $\mu$ thus making them dimensionless

$$
\rho=\frac{\bar{\rho}}{\mu} ; t=\frac{\bar{t}}{\mu} ; g_{A B}=\mu^{2} \bar{g}_{A B}
$$

The TeV brane is located at $\psi=\pi / 2$ and the direction perpendicular to the brane is $\psi=0$. The Planck brane is located at $\rho \cos \psi \sim 1 / \epsilon$. It is outside of the region where the $\epsilon$ expansion is valid. In general, after rescaling the coordinates by $\mu$, the ansatz for the metric is

$$
\begin{aligned}
d s^{2}= & \frac{1}{(1-\epsilon \rho \cos \psi)^{2}}\left[-\left(1-\frac{1}{\rho^{2}}-\sum_{n=1} B_{n}(\rho, \psi) \epsilon^{n}\right) d t^{2}+\left(\frac{\rho^{2}}{\rho^{2}-1}+\sum_{n=1} A_{n}(\rho, \psi) \epsilon^{n}\right) d \rho^{2}\right. \\
& \left.+2 \sum_{n=1} V_{n}(\rho, \psi) \epsilon^{n} d \rho d \psi+\rho^{2}\left(1+\sum_{n=1} U_{n}(\rho, \psi) \epsilon^{n}\right) d \psi^{2}+\rho^{2} \sin ^{2} \psi d \Omega_{2}^{2}\right] .
\end{aligned}
$$

The conformal factor in front of the metric is just the Randall-Sundrum conformal factor and was introduced for convenience. The rescaled equations of motion include the Einstein equations [20]

$$
R_{A B}-\frac{1}{2} R g_{A B}=6 \epsilon^{2} g_{A B}
$$

and the rescaled Israel junction conditions [21] on the TeV brane

$$
2\left(K \gamma_{\mu \nu}-K_{\mu \nu}\right)=6 \epsilon \gamma_{\mu \nu}
$$

The Planck brane is located at a distance $\sim \ell$ from the point mass and therefore the junction condition on the Planck brane can not be evaluated.

Clearly, zeroth order in $\epsilon$ corresponds to vacuum Einstein equations with no branes. The zeroth order solution is therefore the MPS black hole [3]. First order in $\epsilon$ turns the branes on but the cosmological constant of the bulk is still absent. This case corresponds to an empty bulk with a brane and a point like mass on the brane, it is the main subject of this paper. Second order in $\epsilon$ turns on the bulk cosmological constant.

\section{A. First Order in $\epsilon$}

The bulk equations (5) in first order in $\epsilon$ are still the vacuum Einstein equations. However, the solution will be different from the MPS solution since the brane tension is of order $\epsilon$ and it will effect the solution through the junction condition (6).

The ansatz for the metric, up to first order in $\epsilon$, is

$$
\begin{aligned}
d s^{2}=\frac{1}{(1-\epsilon \rho \cos \psi)^{2}} \quad[ & -\left(1-\frac{1}{\rho^{2}}-\epsilon B_{1}\right) d t^{2}+\left(\frac{\rho^{2}}{\rho^{2}-1}+\epsilon A_{1}\right) d \rho^{2} \\
& \left.+2 \epsilon V_{1} d \rho d \psi+\rho^{2}\left(1+\epsilon U_{1}\right) d \psi^{2}+\rho^{2} \sin ^{2} \psi d \Omega_{2}^{2}\right] .
\end{aligned}
$$

We choose to work in a gauge where $t, \theta, \phi$ are kept unchanged, and $g_{\theta \theta}=\rho^{2} \sin ^{2} \psi$. A convenient gauge in the bulk equations is

$$
V_{1}=\frac{2 \rho^{2}-1}{4 \rho} A_{1, \psi}+\frac{\rho^{2} \cot \psi}{2} U_{1, \rho}
$$

The gauge (8) can be achieved using a coordinates transformation of order $\epsilon$

$$
\rho \rightarrow \rho(1-\epsilon F) ; \quad \psi \rightarrow \psi+\epsilon F \tan \psi
$$

The bulk equations are indeed invariant under the transformation (9). However, the junction conditions are not invariant and the function $F(\rho, \psi)$ is not completely arbitrary. It is subject to junction conditions on the brane

$$
F\left(\rho, \frac{\pi}{2}\right)=0 ; F_{, \psi}\left(\rho, \frac{\pi}{2}\right)=0
$$


Using the gauge (8), the bulk equations can be solved in terms of two functions. The gauge function $F(\rho, \psi)$ and the wave function $H(\rho, \psi)$. The solution is

$$
\begin{aligned}
B_{1}= & -\frac{2}{\rho^{3}}\left[6 \rho H_{0}(\rho)-\left(2 \rho^{2}-1\right) H_{0}^{\prime}(\rho)+\rho F(\rho, \psi)\right] \\
A_{1}= & 8 \rho^{3} \cos \psi-\frac{4 \rho^{2}\left(3 H(\rho, \psi)+\tan \psi H_{, \psi}(\rho, \psi)\right)}{\rho^{2}-1}+\frac{12 \rho^{2}\left(\rho^{2}-2\right) H_{0}(\rho)}{\left(\rho^{2}-1\right)^{2}}+\frac{2 \rho\left(2 \rho^{4}-1\right) H_{0}^{\prime}(\rho)}{\left(\rho^{2}-1\right)^{2}} \\
& -\frac{2 \rho^{2}\left(2 \rho^{2}-1\right) H_{0}^{\prime \prime}(\rho)}{\rho^{2}-1}+\frac{2 \rho^{2}\left(\rho^{2}-2\right) F(\rho, \psi)}{\left(\rho^{2}-1\right)^{2}}+\frac{2 \rho^{3} F_{, \rho}(\rho, \psi)}{\rho^{2}-1}, \\
U_{1}= & \frac{2 \tan ^{2} \psi}{\rho}\left\{6 \rho\left[H(\rho, \psi)-H_{0}(\rho)\right]-\left(2 \rho^{2}-1\right)\left[H_{, \rho}(\rho, \psi)-H_{0}^{\prime}(\rho)\right]-\rho F(\rho, \psi)-\rho \cot \psi F_{, \psi}(\rho, \psi)\right\},(1) \\
V_{1}= & -2 \rho^{2}\left(2 \rho^{2}-1\right) \sin \psi-\left(4 \rho^{2}-1\right) \tan \psi H_{0}^{\prime}(\rho)+\rho\left(2 \rho^{2}-1\right) \tan \psi H_{0}^{\prime \prime}(\rho)+2 \rho^{2} \tan \psi H_{, \rho}(\rho, \psi) \\
& +\frac{\rho\left(2 \rho^{2}-1\right) \tan ^{2} \psi H_{, \psi}(\rho, \psi)}{\rho^{2}-1}-\rho^{2} \tan \psi F_{, \rho}(\rho, \psi)+\frac{\rho^{3} F_{, \psi}(\rho, \psi)}{\rho^{2}-1},
\end{aligned}
$$

where $H_{0}(\rho)=H\left(\rho, \frac{\pi}{2}\right)$.

The wave function $H(\rho, \psi)$ is subject to the differential equation

$$
\left(\rho^{2}-1\right)\left(H_{, \rho \rho}-\frac{1}{\rho} H_{, \rho}\right)+H_{, \psi \psi}+2 \frac{\cos ^{2} \psi+1}{\sin \psi \cos \psi} H_{, \psi}=0 .
$$

The general solution can be casted as a combination of Associated Legendre functions in $\rho$ and Hypergeometric functions in $\psi$

$$
\begin{aligned}
H(\rho, \psi) & =\int d \lambda R(\rho ; \lambda) \Psi(\psi ; \lambda) \\
R(\rho ; \lambda) & =\rho \sqrt{\rho^{2}-1}\left[a(\lambda) Q_{(\lambda-1) / 2}^{1}\left(2 \rho^{2}-1\right)+b(\lambda) P_{(\lambda-1) / 2}^{1}\left(2 \rho^{2}-1\right)\right] \\
\Psi(\psi ; \lambda) & =c(\lambda){ }_{2} F_{1}\left(\frac{1-\lambda}{2}, \frac{1+\lambda}{2}, \frac{5}{2}, \sin ^{2} \psi\right)+\frac{d(\lambda)}{\sin ^{3} \psi}{ }_{2} F_{1}\left(\frac{-2-\lambda}{2}, \frac{-2+\lambda}{2},-\frac{1}{2}, \sin ^{2} \psi\right)
\end{aligned}
$$

The first hypergeometric function in Eq. (15) can be expanded by elementary functions as

$$
\begin{aligned}
{ }_{2} F_{1}\left(\frac{1-\lambda}{2}, \frac{1+\lambda}{2}, \frac{5}{2}, \sin ^{2} \psi\right) & =\frac{3[\lambda \cos (\lambda \psi) \sin (2 \psi)-2 \cos (2 \psi) \sin (\lambda \psi)]}{2 \lambda\left(4-\lambda^{2}\right) \sin ^{3} \psi} \\
{ }_{2} F_{1}\left(\frac{1}{2}, \frac{1}{2}, \frac{5}{2}, \sin ^{2} \psi\right) & =\frac{3[\sin (2 \psi)-2 \psi \cos (2 \psi)]}{8 \sin ^{3} \psi} \\
{ }_{2} F_{1}\left(-\frac{1}{2}, \frac{3}{2}, \frac{5}{2}, \sin ^{2} \psi\right) & =\frac{3[4 \psi-\sin (4 \psi)]}{32 \sin ^{3} \psi}
\end{aligned}
$$

The function $H(\rho, \psi)$ is subject to the following boundary conditions.

No black strings. The only source is the (point) mass on the brane at $\psi=\pi / 2$. Therefore, the solution must be regular everywhere. In particular one should eliminate the black string which lies in the bulk on a line perpendicular to the brane at $\psi=0$. The place to look for a string like singularity is the Kretchmann $\operatorname{scalar}\left(R_{A B C D} R^{A B C D}\right)$. The Kretchmann scalar depends only on the combination $3 H+\tan \psi H_{, \psi}$. Using the expansion (15) one finds that

$$
3 \Psi(\psi ; \lambda)+\tan \psi \Psi_{, \psi}(\psi ; \lambda)=\frac{d(\lambda)\left(\lambda^{2}-4\right)}{\psi}+\mathcal{O}\left(\psi^{0}\right) .
$$

A solution free of black strings requires

$$
d(\lambda)=d_{2} \delta(\lambda-2)
$$

The hypergeometric function associated with $d_{2}$ is simply

$$
\left.{ }_{2} F_{1}\left(\frac{-2-\lambda}{2}, \frac{-2+\lambda}{2},-\frac{1}{2}, \sin ^{2} \psi\right)\right|_{\lambda=2}=1 .
$$


Compatibility with Linearized Gravity. At large distances, $\rho \rightarrow \infty$, the solution is given by Linearized Gravity which is an expansion in the mass, $M \sim \mu^{2}$, to first order. Iterating the linearized solution to include higher orders of $M$ one finds that only integer powers of $M$ (even powers of $\mu$ ) enter the metric. Recalling that $\epsilon=\mu / \ell \sim M^{1 / 2}$ and $\rho=\bar{\rho} / \mu \sim M^{-1 / 2}$, it is clear that $\epsilon \rho^{-n} \sim M^{(n+1) / 2}$. Since we allow only for integer powers of $M$, the functions $H(\rho, \psi)$ and $F(\rho, \psi)$ must be odd functions of $\rho$. The function $R(\rho ; \lambda)$ [Eq. (14)] has the same parity as $\rho^{1 \pm \lambda}$. Therefore, the integral over $\lambda$ must turn into a sum over even values of $\lambda$.

Junction conditions on the brane. To first order in $\epsilon$, Israel junction conditions on the TeV brane are simply

$$
B_{1, \psi}\left(\rho, \frac{\pi}{2}\right)=0 ; \quad A_{1, \psi}\left(\rho, \frac{\pi}{2}\right)=0 ; \quad V_{1}\left(\rho, \frac{\pi}{2}\right)=0
$$

Using the function (13), these conditions imply that

$$
\int d \lambda R(\rho ; \lambda) c(\lambda) \cos \left(\frac{\pi \lambda}{2}\right)=-\frac{2}{3}\left(\rho^{3}-\rho\right)
$$

The asymptotic behavior of the Legendre functions of the first kind is: $\rho \sqrt{\rho^{2}-1} P_{(\lambda-1) / 2}^{1}\left(2 \rho^{2}-1\right) \sim \rho^{1+\lambda}$. Therefore, to prevent higher powers of $\rho$, one must eliminate the Legendre functions of the first kind except for $\lambda=2$ which is the only function that can generate an asymptotic behavior $\rho^{3}$. In particular

$$
b(\lambda)=b_{2} \delta(\lambda-2)
$$

Still, the Legendre function of the first kind, with $\lambda=2$, includes a term $(\log \rho) / \rho$ in the asymptotic expansion. In order to eliminate the logarithmic term from the condition (21) one must use the combination

$$
\left[P_{(\lambda-1) / 2}^{1}\left(2 \rho^{2}-1\right)+\frac{4}{\pi^{2}} \frac{\partial}{\partial \lambda} Q_{(\lambda-1) / 2}^{1}\left(2 \rho^{2}-1\right)\right]_{\lambda=2} .
$$

This combination can be obtained by setting

$$
a(\lambda)=\sum_{n=0} a_{2 n} \delta(\lambda-2 n)-\frac{4}{\pi^{2}} b_{2} \delta^{\prime}(\lambda-2) .
$$

Asymptotic boundary conditions. One would like to impose boundary conditions at $\rho \rightarrow \infty$. The problem is that the $\epsilon$ expansion is valid only for $1 \leq \rho \ll 1 / \epsilon$ (which corresponds to $\mu \leq \bar{\rho} \ll \ell$ ). However, the asymptotic form of the metric is given by the linearized solution. The later is valid for $1 \ll \rho(\mu \ll \bar{\rho})$. As long as $\epsilon \ll 1$, there is an intermediate region $1 \ll \rho \ll 1 / \epsilon$ where both the $\epsilon$ solution and the linearized solution are valid. Therefore, the large $\rho$ limit of the $\epsilon$ solution should coincide with first order in $\epsilon$ of the linearized solution. The appropriate asymptotic boundary condition is

$$
\lim _{\rho \rightarrow \infty} \rho\left(g_{A B}^{\epsilon}-g_{A B}^{L}\right)=0
$$

To summarize, collecting Eqs.(13), (18), (22), and (24), the function $H(\rho, \psi)$ is

$$
\begin{aligned}
H(\rho, \psi)= & \rho \sqrt{\rho^{2}-1}\left[a_{0} Q_{-1 / 2}^{1}\left(2 \rho^{2}-1\right) \frac{3[\sin (2 \psi)-2 \psi \cos (2 \psi)]}{8 \sin ^{3} \psi}\right. \\
& +Q_{1 / 2}^{1}\left(2 \rho^{2}-1\right)\left(a_{2} \frac{3[4 \psi-\sin (4 \psi)]}{32 \sin ^{3} \psi}+\frac{d_{2}}{\sin ^{3} \psi}-b_{2} \frac{3[8 \psi+4 \psi \cos (4 \psi)-3 \sin (4 \psi)]}{32 \pi^{2} \sin ^{3} \psi}\right) \\
& +b_{2}\left(P_{1 / 2}^{1}\left(2 \rho^{2}-1\right)+\left.\frac{2}{\pi^{2}} \frac{\partial Q_{n-1 / 2}^{1}\left(2 \rho^{2}-1\right)}{\partial n}\right|_{n=1}\right) \frac{3[4 \psi-\sin (4 \psi)]}{32 \sin ^{3} \psi} \\
& \left.+\sum_{n=2} a_{2 n} Q_{n-1 / 2}^{1}\left(2 \rho^{2}-1\right) \frac{3[n \cos (2 n \psi) \sin (2 \psi)-\cos (2 \psi) \sin (2 n \psi)]}{8 n\left(1-n^{2}\right) \sin ^{3} \psi}\right] .
\end{aligned}
$$

It includes a discrete set of parameters; $d_{2}, b_{2}$, and $a_{2 n}(n=0,1, .$.$) . These parameters should be fixed by the$ asymptotic boundary condition (25) and the junction condition (21). 
So, we turn first to the asymptotic behavior of the solution. The linearized solution appears in appendix $\AA$ Expansion of the linearized solution to first order in $\epsilon$ appears in Eqs. A23). The latter imply

$$
\begin{aligned}
B_{1}^{L} & =\frac{1}{\rho}\left[\cos \psi+\frac{\psi}{\sin \psi}\right], \\
A_{1}^{L} & =\frac{1}{\rho}\left[-\frac{5}{4} \cos \psi-\frac{3}{2} \psi \sin \psi+\frac{\psi}{4 \sin \psi}-\rho \cos \psi W_{1, \rho}\right] \\
U_{1}^{L} & =\frac{1}{\rho}\left[\frac{11}{8} \cos \psi+\frac{5}{8} \cos (3 \psi)-\frac{2 \psi}{\sin \psi}+\frac{3}{2} \psi \sin \psi+\sin \psi W_{1, \psi}\right], \\
V_{1}^{L} & =\frac{\cos ^{2} \psi(2-5 \cos (2 \psi))}{8 \sin \psi}+\frac{3 \psi \cos (3 \psi)}{8 \sin ^{2} \psi}+\frac{1}{2} \rho \sin \psi W_{1, \rho}-\frac{1}{2} \cos \psi W_{1, \psi} .
\end{aligned}
$$

The function $W_{1}(\rho, \psi)$ is arbitrary, but is subject to the boundary conditions

$$
W_{1}\left(\rho, \frac{\pi}{2}\right)=0 ; W_{1, \psi}\left(\rho, \frac{\pi}{2}\right)=\frac{\pi}{4} .
$$

To simplify the analysis [to avoid the function $W_{1}(\rho, \psi)$ ], one should look at the following combinations; $B_{1}, C_{1}=$ $\left(A_{1} /(\cos \psi)\right)_{, \psi}+\left(\rho U_{1} /(\sin \psi)\right)_{, \rho}, D_{1}=\sin ^{2} \psi A_{1}+\cos ^{2} \psi U_{1}+V_{1} \sin (2 \psi) / \rho$, and $\left.U_{1}^{L}\right|_{\psi=\pi / 2}$.

$$
\begin{aligned}
B_{1}^{L} & =-\frac{1}{\rho}\left[\cos \psi+\frac{\psi}{\sin \psi}\right], \\
C_{1}^{L} & =\frac{1}{\rho \sin ^{2}(2 \psi)}\left[2 \psi \cos (2 \psi)-\sin (2 \psi)+\frac{3}{4} \sin (4 \psi)-3 \psi\right], \\
D_{1}^{L} & =\frac{5}{16 \rho \sin \psi}[\sin (4 \psi)-4 \psi], \\
\left.U_{1}^{L}\right|_{\psi=\pi / 2} & =0 .
\end{aligned}
$$

For the purpose of comparing the $\epsilon$ solution and the linearized solution, the $\epsilon$ solution should be expanded in inverse powers of $\rho$ only up to order $\epsilon \rho^{-1} \sim M$. Expanding the solution (11] 26) to first order in $\rho^{-1}$, and casting the gauge function as $F(\rho, \psi)=\rho F_{1}(\psi)+F_{2}(\psi) / \rho$, the asymptotic behavior of the $\epsilon$ solution is

$$
\begin{aligned}
B_{1}^{\epsilon} & =\frac{1}{\rho}\left[\frac{3 \pi^{2}}{4} a_{0}-2 F_{1}(\psi)\right], \\
C_{1}^{\epsilon} \quad & =\frac{1}{\rho \sin ^{2}(2 \psi)}\left[8 \sin ^{3} \psi F_{1}(\psi)+8 \cos \psi \sin ^{2} \psi F_{1}^{\prime}(\psi)\right. \\
& \left.-3 \pi a_{0}\left(3 \psi \cos (2 \psi)-3 \sin \psi \cos \psi+\pi \sin ^{3} \psi\right)+\frac{9 b_{2}}{4 \pi}(\sin (4 \psi)-4 \psi)\right], \\
D_{1}^{\epsilon} \quad & =\frac{4}{\pi} \rho \cos \psi \sin ^{2} \psi\left[\pi-3 b_{2}\right]+\frac{1}{\rho}\left[\frac{3 \pi a_{0}}{4 \sin \psi}\left(\psi-2 \psi \cos (2 \psi)+\sin \psi \cos \psi-\pi \sin ^{3} \psi\right)\right. \\
& \left.+\frac{3 b_{2}}{8 \pi}\left(\cos \psi+5 \cos (3 \psi)-\frac{6 \psi}{\sin \psi}\right)+2 \sin ^{2} \psi F_{1}(\psi)+\sin (2 \psi) F_{1}^{\prime}(\psi)\right], \\
\left.U_{1}^{\epsilon}\right|_{\psi=\pi / 2} & =\rho\left[\frac{3 \pi^{2} a_{0}}{8}+F_{1}^{\prime \prime}(\pi / 2)\right]+\frac{1}{\rho}\left[-\frac{3 \pi^{2} a_{0}}{32}-\frac{27 \pi^{2} a_{2}}{64}-\frac{9 \pi d_{2}}{4}-\frac{45 b_{2}}{16}+F_{2}^{\prime \prime}(\pi / 2)\right] .
\end{aligned}
$$

Comparing $B_{1}^{L}$ and $B_{1}^{\epsilon}$ [Eqs.(29a), (30a)] and using the junction condition for $F(\rho, \psi)$ [Eq. (10)] one finds that

$$
a_{0}=-\frac{2}{3 \pi} ; F_{1}(\psi)=\frac{1}{2}\left(\cos \psi+\frac{\psi}{\sin \psi}-\frac{\pi}{2}\right) .
$$

Going next to $D_{1}^{L}$ and $D_{1}^{\epsilon}$ [Eqs. (29c), (30c)]. One finds that

$$
b_{2}=\frac{\pi}{3} .
$$

Finally, Eqs.(29d) and (30d) imply that

$$
F_{2}^{\prime \prime}\left(\frac{\pi}{2}\right)=\frac{\pi}{64}\left(56+144 d_{2}+27 \pi a_{2}\right) .
$$


The rest of the set $a_{2 n}$ can be evaluated by means of the junction condition on the brane (21)

$$
\begin{aligned}
-\frac{2}{3}\left(\rho^{3}-\rho\right)= & \sum_{n=1} a_{2 n}(-1)^{n} \rho \sqrt{\rho^{2}-1} Q_{n-1 / 2}^{1}\left(2 \rho^{2}-1\right)-\frac{2}{3 \pi} \rho \sqrt{\rho^{2}-1} Q_{-1 / 2}^{1}\left(2 \rho^{2}-1\right) \\
& -\frac{\pi}{3} \rho \sqrt{\rho^{2}-1}\left(P_{1 / 2}^{1}\left(2 \rho^{2}-1\right)+\left.\frac{2}{\pi^{2}} \frac{\partial Q_{n-1 / 2}^{1}\left(2 \rho^{2}-1\right)}{\partial n}\right|_{n=1}\right) .
\end{aligned}
$$

To simplify Eq.(34), we set $z=2 \rho^{2}-1$ and divide the equation by $\rho \sqrt{\rho^{2}-1}$

$$
\sum_{n=1} a_{2 n}(-1)^{n} Q_{n-1 / 2}^{1}(z)=-\frac{2}{3} \sqrt{\frac{z-1}{2}}+\frac{2}{3 \pi} Q_{-1 / 2}^{1}(z)+\frac{\pi}{3} P_{1 / 2}^{1}(z)+\left.\frac{2}{3 \pi} \frac{\partial}{\partial n} Q_{n-1 / 2}^{1}(z)\right|_{n=1} .
$$

according to a theorem by Banerjee [22] the toroidal functions $Q_{n-1 / 2}^{1}(z)$ form a complete set on $(1, \infty)$. The function on the right hand side goes to zero as $\rho \rightarrow \infty$. Therefore it can be expanded in a series of toroidal functions of the second kind i.e. the left hand side. The prescription given by Banerjee [22] is quite complicated and we will not follow it here. Alternatively, the coefficients can be found recursively from the asymptotic expansion of Eq. (34)

$$
a_{2}=-\frac{1}{3 \pi} ; a_{4}=\frac{4}{9 \pi} ; a_{6}=\frac{41}{21 \pi} \ldots
$$

A single parameter $d_{2}$ remains undetermined.

\section{THE BLACK HOLE HORIZON}

The existence of an event horizon is a delicate issue. It is clear that before turning on the brane $(\epsilon=0)$, there exists a single horizon at $\rho_{H}=1$. There is no guarantee that first order in $\epsilon$ will not ruin the horizon.

We assume that the new horizon is located at $\rho_{H}=1+\epsilon \zeta$. We expand $g_{t t}(\rho=1+\epsilon \zeta)=0$ to first order in $\epsilon$ to get

$$
2 \epsilon \zeta+2 \epsilon\left[F(1, \psi)+1+\beta \log \left|\frac{\epsilon \zeta}{8}\right|\right]=0
$$

with $\beta=\left(6 d_{2}+1\right) / 8$ depend on the parameter $d_{2}$.

The gauge function $F(1, \psi)$ introduces a $\psi$ dependence. But, there is still a gauge freedom to choose $F(1, \psi)=0$ which is in complete agreement with the junction conditions (10) and (3133). We choose the gauge $F(1, \psi)=0$ from now on.

The logarithmic term comes from the associated Legendre functions of the second kind which close to 1 behave like

$$
\rho \sqrt{\rho^{2}-1} Q_{n-1 / 2}^{1}\left(2 \rho^{2}-1\right) \sim-\frac{1}{2}+\frac{\left(4 n^{2}-1\right)(\rho-1)}{4}\left[-\log |\rho-1|+C_{n}\right]+\mathcal{O}\left((\rho-1)^{2}\right) .
$$

This is the first indication that the solution (26) is not regular on the horizon. The second indication comes from the surface gravity. The surface gravity calculated from the solution (26) is not constant on the horizon. According to [19] this shows that the horizon is singular.

There are few possible explanations. One option is that the configuration indeed becomes singular already at first order in $\epsilon$. This means that there are no small black holes in Randall-Sundrum scenario. Another possibility is that although the $\epsilon$ solution is valid close to the horizon it does not converge on the horizon. This can be seen if one tries to expand $g_{t t}^{-1}$ in $\epsilon$, clearly, the expansion does not converge on the horizon $\left(g_{t t}=0\right)$.

The regularity of the horizon is still under study. The detailed discussion of this issue is left for a future publication.

\section{SUMMARY}

Randall-Sundrum 1 model makes the gravitational interaction strong on small scales and might allow for small black holes on the $\mathrm{TeV}$ brane. Interesting phenomena might arise in the presence of TeV scale black holes. Especially, the way such primordial black-holes accrete or radiate matter when immersed in a hot plasma would affect the cosmological 
evolution of the universe. The behavior of a growing or evaporating black hole is related to its thermodynamic properties. Thus the study of its horizon is required.

The purpose of this paper is to introduce an approximation method for studying the horizon of a small black hole in the Randall-Sundrum I scenario. 'Small' black hole means that the five dimensional Schwarzschild radius of the black hole is smaller than the curvature length of the bulk AdS manifold. The method suggested in this paper is to expand the metric in the dimensionless parameter $\epsilon=\sqrt{G_{5} M} / \ell$, and to fix the asymptotic behavior using the weak field approximation (linearized gravity). Zeroth order in $\epsilon$ is just a Myers-Perry flat background black hole. First order in $\epsilon$ corresponds to the presence of a brane with brane tension which is of order $\epsilon$ in a flat bulk. Second order in $\epsilon$ includes also the bulk cosmological constant which is of order $\epsilon^{2}$.

We fix the asymptotic behavior in the following way. The $\epsilon$ solution is valid in the region $r \ll \ell$. It includes the region close to the horizon which is essential for horizon studies. However, it is not valid in the asymptotic region $r \rightarrow \infty$ and one is unable to identify the mass or satisfy the junction conditions on the second brane (at distance $\sim \ell$ ). In order to overcome these subtleties we use the linearized solution as an asymptotic boundary condition. Linearized gravity is valid in the region $r \gg \mu$. We are interested in the case $\mu \ll \ell$, therefore, there is an intermediate region $\mu \ll r \ll \ell$ where the $\epsilon$ solution and the linearized solution can be matched. In this intermediate range we take the large distance behavior of the $\epsilon$ solution and match it to the short distance behavior of the linearized solution. As a result, the long range characteristics (mass identification and second brane conditions) are introduced into the $\epsilon$ solution.

We should mention that the method of expanding in $\epsilon$ and comparing with the linearized solution as an asymptotic condition is applicable to other scenarios as well. For example: Randall-Sundrum single brane scenario, de Sitter bulk (expanding in the ratio between the Schwarzschild radius and the curvature length), compact extra dimension (expanding in the ratio between the Schwarzschild radius and the compactification radius), etc.

In this paper we have found a solution up to first order in $\epsilon$. We require that the curvature singularity resides only on the brane. No black strings are associated with our solution. The mass of the object consists of matter completely confined to the brane.

Although the $\epsilon$ solution is valid in the region close to the horizon, it is not obvious that the new horizon is regular. The $\epsilon$ solution acquires a null surface defined by $g_{t t}=0$. The normal vector is null and the static Killing vector is null i.e. the surface is a Killing Horizon. But, the surface gravity of the horizon is not a constant which indicates that the horizon is singular [19]. This leaves us with the following possibilities; (1) The horizon is singular. Which means that there are no small black holes in Randall-Sundrum scenario. (2) The $\epsilon$ expansion does not converge on the horizon. It is valid only at a distance $\epsilon$ away from the horizon, and therefore one cannot use it to calculate the surface gravity. (3) The boundary conditions should be changed thus changing the entire solution.

The issue of the regularity of the horizon is under study and the detailed discussion is left for a future publication.

\section{Acknowledgments}

This work is supported in part by the U.S. Department of Energy Grant No. DE-FG02-84ER40153. We thank Philip Argyres and Cenalo Vaz for fruitful discussions.

\section{APPENDIX A: LINEARIZED GRAVITY}

\section{Randall-Sundrum I Background}

RSI scenario consists of two flat 3-branes embedded in five dimensional AdS bulk. The equations of motion in the bulk are the five dimensional Einstein equations [20]

$$
R_{A B}-\frac{1}{2} R g_{A B}=8 \pi G_{5} T_{A B}=\frac{6}{\ell^{2}} g_{A B} .
$$

With an additional $Z_{2}$ symmetry with respect to the brane, the Israel junction conditions are

$$
2\left(K \gamma_{\mu \nu}-K_{\mu \nu}\right)=8 \pi G_{5} S_{\mu \nu}=\frac{ \pm 6}{\ell} \gamma_{\mu \nu} .
$$

The upper (lower) sign corresponds to the negative (positive) tension brane.

Parameterizing the bulk with the coordinates $y^{A}=\left(x^{\mu}, w\right)$, where $w$ is the coordinate perpendicular to the brane, a conformally flat ansatz for the metric is

$$
d s_{5}^{2}=a^{2}(w) \eta_{A B} d y^{A} d y^{B}
$$


We choose a coordinate system in which the TeV brane (with negative tension) is located at $w=0$, while the Planck brane (positive tension) is at $|w|=\ell(1-\lambda) . \lambda$ is a very small number. It is the ratio between the TeV scale and the Planck scale, namely $\lambda \sim 10^{-19}$. The conformal factor in (A33) is $a^{2}(w)=\left(\frac{\ell}{\ell-|w|}\right)^{2}$. It has the value 1 on the $\mathrm{TeV}$ brane (at $w=0$ ) and the value $\lambda^{-2}$ on the Planck brane. In this scenario the standard model particles are assumed to be confined by some mechanism to the TeV brane.

Let us assume now that there is a matter distribution on the brane such that it is confined to a small region on the brane at $w=0$, therefore, the energy momentum tensor is non vanishing only at $\left(r<r_{0}, w=0\right)$. However, if we are interested in the solution far from this region, we can use the linearized version of Eqs. A1 A2 . The perturbed metric is given by

$$
d s_{5}^{2}=a^{2}(w)\left[\eta_{A B}+h_{A B}\left(x^{\mu}, w\right)\right] d y^{A} d y^{B} .
$$

The perturbation $h_{A B}$ is assumed to be small. The source for the perturbation is the matter on the brane, characterized by the energy momentum tensor, $T_{\mu \nu}$. The total energy momentum tensor on the brane (which enters Israel junction condition (A2)) is $S_{\mu \nu}=\sigma \gamma_{\mu \nu}+T_{\mu \nu}$.

\section{Conserved Momentum}

The definition of a conserved momentum is usually done in an asymptotically Minkowski $n+1$ dimensional spacetime as $P^{A}=\int d^{n} x T^{A 0}$. It is associated with energy momentum conservation $T_{, B}^{A B}=0$, where the use of ordinary derivative instead of covariant derivative is essential.

In RS models, one deals with asymptotically conformal Minkowski space-time, and the energy-momentum conservation condition is $T_{; B}^{A B}=0$. One can verify, however, that the energy momentum conservation condition can be written as

$$
T_{A ; B}^{B}=\frac{1}{\sqrt{-g}}\left(\sqrt{-g} T_{A}^{B}\right)_{, B}-\frac{a^{\prime}(w)}{a(w)} T_{B}^{B} \delta_{A w}=0 .
$$

Therefore, since the conformal factor $a(w)$ depends only on the fifth coordinate, one is able to define a conserved four momentum $P_{\mu}^{(5)}=\int d^{3} x d w \sqrt{-g} T_{\mu}^{0}$. One can verify that the momentum is conserved using Eq. A5

$$
\dot{P}_{\mu}^{(5)}=-\int d^{3} x d w\left[\left(\sqrt{-g} T_{\mu}^{i}\right)_{, i}+\left(\sqrt{-g} T_{\mu}^{w}\right)_{, w}\right]=-\lim _{r \rightarrow \infty} \int d w d \Omega r^{2} n_{i} \sqrt{-g} T_{\mu}^{i}-\int d^{3} x d w\left(\sqrt{-g} T_{\mu}^{w}\right)_{, w} .
$$

The first integral on the right hand side vanishes provided the matter is confined to a small region. The second integral on the right hand side is treated differently in different scenarios; in RSII, with a single brane, the $w$ integration is over the interval $(-\infty, \infty)$ and vanishes provided the matter is confined to the brane and the energy-momentum component $T_{\mu}^{w}$ is continuous. In RSI with two branes the only requirement is the continuity of $T_{\mu}^{w}$.

It is worth noting that if the energy-momentum tensor is traceless (for example four dimensional Maxwell field), then one is able to define a conserved five momentum $P_{A}^{(5)}=\int d^{3} x d w \sqrt{-g} T_{A}^{0}$. In other words, the presence of the trace brakes a symmetry and therefore the theory has only a conserved four momentum.

The solution to Eq. A5 is given by $\sqrt{-g} T_{\mu}^{B}=\partial_{D} Q_{\mu}^{D B}$, with $Q_{\mu}^{D B}=-Q_{\mu}^{B D}$. The exact form of $Q_{\mu}^{D B}$ can be calculated from the linearized Einstein equations. The conserved momentum can be calculated using

$$
P_{\mu}^{(5)}=\int d^{3} x d w \partial_{w} Q_{\mu}^{w 0}+\int d \Omega d w r^{2} n_{i} Q_{\mu}^{i 0} .
$$

The first integral has contributions only from the discontinuities of $Q_{\mu}^{w 0}$. This can be evaluated using the linearized junction conditions and turns out to be exactly the four momentum of matter confined to the branes

$$
\int d^{3} x d w \partial_{w} Q_{\mu}^{w 0}=\left.\int d^{3} x T_{\mu}^{0}\right|_{\text {branes }}=P_{\mu}^{(4)} .
$$

The five dimensional momentum can be summarized as

$$
P_{\mu}^{(5)}=P_{\mu}^{(4)}+\lim _{r \rightarrow \infty} \frac{1}{16 \pi G_{5}} \int d \Omega d w a^{3}(w) r^{2} n^{i}\left(-h_{\mu i, 0}-h_{\mu, i}^{0}+\eta_{\mu i} h_{, \nu}^{0 \nu}-\delta_{\mu}^{0} h_{i, j}^{j}+\delta_{\mu}^{0} h_{B, i}^{B}+\eta_{\mu i} h_{B, 0}^{B}\right) .
$$


The energy $P_{0}$ is given by

$$
P_{0}^{(5)}=P_{0}^{(4)}+\lim _{r \rightarrow \infty} \frac{1}{16 \pi G_{5}} \int d \Omega d w a^{3}(w) r^{2} n^{i}\left[-h_{0 i, 0}-h_{i, j}^{j}+h_{k, i}^{k}+h_{w w, i}\right] .
$$

If the only sources for the perturbation reside on the branes, then the five dimensional and the four dimensional momenta are equal, and the integrals in Eqs. A910 must vanish.

\section{Linearized Solution}

Choosing the gauge $g_{\theta \theta}=a^{2}(w) r^{2}$, the metric perturbation caused by a single point-like mass, $M$, located on the $\mathrm{TeV}$ brane is given in radial coordinates as

$$
\begin{aligned}
h_{t t}(r, w) & =\frac{2 C}{r}+\frac{2 G_{5} M}{3 r \ell}\left[2 \tilde{F}_{1}(\alpha, \beta)\right]+2 \frac{W(r, w)}{\ell-w}, \\
h_{r r}(r, w) & =\frac{2 C}{r}+\frac{2 G_{5} M}{3 r \ell}\left[\tilde{F}_{1}(\alpha, \beta)-\alpha \tilde{F}_{1, \alpha}\right]+\frac{2 r W_{, r}(r, w)}{\ell-w}, \\
h_{\theta \theta}(r, w) & =0, \\
h_{w w}(r, w) & =-2\left(\frac{W(r, w)}{\ell-w}+W_{, w}(r, w)\right), \\
h_{r w}(r, w) & =\frac{G_{5} M}{3 \ell^{2}} \tilde{F}_{2}-W_{, r}(r, w)+\frac{r}{\ell-w}\left(\frac{W(r, w)}{\ell-w}+W_{, w}(r, w)\right) .
\end{aligned}
$$

The term $\frac{2 C}{r}$ is the contribution of the zero-mode. The constant $C$ cannot be determined by the bulk equations nor by the junction conditions. The only way to determine $C$ would be through the definition of the total mass of the configuration given by Eq. A10).

The terms with $\tilde{F}_{1}, \tilde{F}_{2}$ are the massive tachyonic modes of the linearized equations. These functions depend on the dimensionless variables $\alpha=r / \ell, \beta=(\ell-w) / \ell$, and are given by

$$
\begin{aligned}
& \tilde{F}_{1}(\alpha, \beta)=\frac{2 \beta^{2}}{\pi} \int_{0}^{\infty} d z \sin (z \alpha) \frac{K_{1}(z \lambda) I_{2}(z \beta)+I_{1}(z \lambda) K_{2}(z \beta)}{K_{1}(z \lambda) I_{1}(z)-I_{1}(z \lambda) K_{1}(z)} \\
& \tilde{F}_{2}(\alpha, \beta)=\frac{2 \beta^{2}}{\pi} \int_{0}^{\infty} d z\left[\sin (z \alpha)-\frac{\sin (z \alpha)}{z^{2} \alpha^{2}}+\frac{\cos (z \alpha)}{z \alpha}\right] z \frac{K_{1}(z \lambda) I_{1}(z \beta)-I_{1}(z \lambda) K_{1}(z \beta)}{K_{1}(z \lambda) I_{1}(z)-I_{1}(z \lambda) K_{1}(z)} .
\end{aligned}
$$

$W(r, w)$ is a gauge function which cannot be determined by the bulk equations. It represents a coordinates transformation of the form

$$
r \rightarrow r ; \quad w \rightarrow w+W(r, w)
$$

It is subject to the following boundary conditions

$$
\begin{array}{rll}
W(r, w=0)=\frac{G_{5} M}{3 r} & ; & W_{, w}(r, w=0)=-\frac{G_{5} M}{3 r \ell} \\
W(r, w=\ell(1-\lambda))=0 & ; \quad W_{, w}(r, w=\ell(1-\lambda))=0
\end{array}
$$

On the TeV brane $(w=0) W(r, 0)$ behaves like a massless four dimensional scalar mode, and is called "the radion".

The definition of the mass and the hierarchy should be seen at the asymptotic region $r \gg \ell$. The asymptotic expansion of the functions $\tilde{F}_{1}, \tilde{F}_{2}$ is given by

$$
\begin{aligned}
& \tilde{F}_{1}(\alpha \gg 1, \beta) \sim \frac{2 \lambda^{2}}{1-\lambda^{2}}, \\
& \tilde{F}_{2}(\alpha \gg 1, \beta) \sim-\frac{\beta\left(\beta^{2}-\lambda^{2}\right)}{\alpha^{2}\left(1-\lambda^{2}\right)} .
\end{aligned}
$$

Using Eq. A10 and assuming that there are no other sources besides the point mass on the TeV brane, one can fix the constant $C$ in the zero mode $C=-\frac{2 G_{5} M \lambda^{2}}{3 \ell\left(1-\lambda^{2}\right)}$. The zero mode tends to be repulsive. 
The gauge invariant force acting on a slowly moving test particle is given by

$$
F_{r}=\frac{1}{2} h_{t t, r}+\frac{1}{\ell-w} h_{r w}
$$

The gravitational potential along the $\mathrm{TeV}$ brane at distances much larger than the curvature length $r \gg \ell$ is given by

$$
V=-\frac{2 G_{5} M \lambda^{2}}{3 r \ell\left(1-\lambda^{2}\right)}-\frac{G_{5} M}{3 r \ell}
$$

The first term is the contribution of the zero-mode and the massive modes. These sum up together to give the correct hierarchy with $G_{4}=\frac{2 G_{5} \lambda^{2}}{3 \ell\left(1-\lambda^{2}\right)}$.

The last term in Eq. A17 is the contribution of the radion and is clearly destroying the hierarchy and producing a long range strong $(\mathrm{TeV})$ gravitational attraction. In order to save the hierarchy it is necessary to include a stabilization mechanism that removes the radion at large distances. A stabilization mechanism was suggested in 23]. It introduces a bulk scalar field which is coupled to the radion by some potential and cause the radion to acquire mass. Thus, the radion acquires a gravitational potential of the form $e^{-m_{r} r} / r$ and does not contribute at distances $r \gg \frac{1}{m_{r}} \log \left(\frac{1}{\lambda^{2}}\right)$. In general, the introduction of a stabilizing bulk scalar field will change the RSI background, namely the conformal factor, and might have an impact on the graviton modes as well.

In this paper we are interested in the short range region $\mu<r<\ell$. Therefore, assuming that the radion becomes massive by some mechanism but is still light compared to the curvature length $m_{r}<\frac{1}{\ell} \sim 10-100 \mathrm{GeV}$, we can assume that in the region $r<\ell$ the above discussion is valid and the metric for the linearized solution is given by Eqs. A11. In addition, we assume that the stabilizing bulk scalar field is massive enough not to interfere in the region $\mu<r<\ell$, thus assuming $M_{\Phi}>\frac{1}{\mu} \sim 1 T e V$.

The asymptotic expansion of the functions $\tilde{F}_{1}, \tilde{F}_{2}$ in the region $r \ll \ell$ is given by

$$
\begin{aligned}
& \tilde{F}_{1}(\alpha \ll 1, \beta) \sim \frac{2 \alpha \beta^{3 / 2}}{\pi\left(\alpha^{2}+(1-\beta)^{2}\right)}-\frac{3(5-\beta) \sqrt{\beta}}{4 \pi} \arctan \left(\frac{\alpha}{1-\beta}\right) \\
& \tilde{F}_{2}(\alpha \ll 1, \beta) \sim \frac{4 \alpha(1-\beta) \beta^{3 / 2}}{\pi\left(\alpha^{2}+(1-\beta)^{2}\right)^{2}}+\frac{\left(3 \beta^{2}+2 \beta+3\right) \sqrt{\beta}}{4 \pi \alpha}\left[\frac{1-\beta}{\alpha^{2}+(1-\beta)^{2}}-\frac{1}{\alpha} \arctan \left(\frac{\alpha}{1-\beta}\right)\right]
\end{aligned}
$$

Using these expansions, and also expanding the gauge function as

$$
W(r, w)=\frac{\mu^{2}}{2}\left[W_{0}(r, w)+\frac{1}{\ell} W_{1}(r, w)\right]
$$

one can write the expanded form of the metric A11, to first order in $1 / \ell$.

$$
\begin{aligned}
h_{t t}(r, w)= & \frac{\mu^{2}}{r^{2}+w^{2}}+\frac{\mu^{2}}{\ell}\left[-\frac{3}{2 r} \arctan \left(\frac{r}{w}\right)-\frac{3 w}{2\left(r^{2}+w^{2}\right)}+W_{0}(r, w)\right], \\
h_{r r}(r, w)= & \frac{\mu^{2} r^{2}}{\left(r^{2}+w^{2}\right)^{2}}+\frac{\mu^{2}}{\ell}\left[-\frac{3}{4 r} \arctan \left(\frac{r}{w}\right)+\frac{3 w\left(w^{2}-r^{2}\right)}{4\left(r^{2}+w^{2}\right)^{2}}+r W_{0, r}(r, w)\right], \\
h_{\theta \theta}(r, w)= & 0, \\
h_{w w}(r, w)= & -\mu^{2} W_{0, w}(r, w)-\frac{\mu^{2}}{\ell}\left[W_{0}(r, w)+W_{1, w}(r, w)\right], \\
h_{r w}(r, w)= & \mu^{2}\left[-\frac{1}{4 r^{2}} \arctan \left(\frac{r}{w}\right)+\frac{w\left(3 r^{2}+w^{2}\right)}{4 r\left(r^{2}+w^{2}\right)^{2}}-\frac{1}{2} W_{0, r}(r, w)\right] \\
& +\frac{\mu^{2}}{\ell}\left[\frac{3 w}{8 r^{2}} \arctan \left(\frac{r}{w}\right)-\frac{3 w^{2}\left(3 r^{2}+w^{2}\right)}{8 r\left(r^{2}+w^{2}\right)^{2}}+\frac{r}{2} W_{0, w}(r, w)-\frac{1}{2} W_{1, r}(r, w)\right] .
\end{aligned}
$$

The boundary conditions for $W(r, w)$ are

$$
\begin{aligned}
& W_{0}(r, 0)=\frac{\pi}{4 r} \quad ; \quad W_{1}(r, 0)=0 ; \\
& W_{0, w}(r, 0)=0 \quad ; \quad W_{1, w}(r, 0)=-\frac{\pi}{4 r} .
\end{aligned}
$$


In order to compare the linearized solution (A20) and the $\epsilon$ expansion (11), one has to perform a coordinate transformation to rescale the linearized-metric (A20)

$$
\rho=\frac{\sqrt{r^{2}+w^{2}}}{\mu} ; \psi=\arctan \left(\frac{r}{w}\right) ; g_{A B} \rightarrow \frac{1}{\mu^{2}} g_{A B} ; \epsilon=\frac{\mu}{\ell} .
$$

After transformation (A22) the linearized metric A20 is

$$
\begin{aligned}
(1-\epsilon \rho \cos \psi)^{2} g_{t t}^{L} & =-1+\frac{1}{\rho^{2}}-\frac{\epsilon}{\rho}\left[\cos \psi+\frac{\psi}{\sin \psi}\right] \\
(1-\epsilon \rho \cos \psi)^{2} g_{\rho \rho}^{L} & =1+\frac{1}{\rho^{2}}+\frac{\epsilon}{\rho}\left[-\frac{5}{4} \cos \psi-\frac{3}{2} \psi \sin \psi+\frac{\psi}{4 \sin \psi}-\rho \cos \psi W_{1, \rho}\right] \\
(1-\epsilon \rho \cos \psi)^{2} g_{\psi \psi}^{L} & =\rho^{2}+\epsilon \rho\left[\frac{11}{8} \cos \psi+\frac{5}{8} \cos (3 \psi)-\frac{2 \psi}{\sin \psi}+\frac{3}{2} \psi \sin \psi+\sin \psi W_{1, \psi}\right] \\
(1-\epsilon \rho \cos \psi)^{2} g_{\rho \psi}^{L} & =\epsilon\left[\frac{\cos ^{2} \psi(2-5 \cos (2 \psi))}{8 \sin \psi}+\frac{3 \psi \cos (3 \psi)}{8 \sin ^{2} \psi}+\frac{1}{2} \rho \sin \psi W_{1, \rho}-\frac{1}{2} \cos \psi W_{1, \psi}\right] \\
(1-\epsilon \rho \cos \psi)^{2} g_{\theta \theta}^{L} & =\rho^{2} \sin ^{2} \psi
\end{aligned}
$$

The first term of the gauge function $W(\rho, \psi)$ A19 ixed by setting $\epsilon=0$

$$
W_{0}(\rho, \psi)=\frac{1}{2 \mu \rho}\left(\cos \psi+\frac{\psi}{\sin \psi}\right) \text {. }
$$

The second part of the function $W(\rho, \psi)$ (A19) is yet undetermined, but is subject to the boundary conditions

$$
W_{1}\left(\rho, \frac{\pi}{2}\right)=0 ; W_{1, \psi}\left(\rho, \frac{\pi}{2}\right)=\frac{\pi}{4}
$$

[1] L. Randall and R. Sundrum, Phys. Rev. Lett. 83:3370-3 (1999).

[2] L. Randall and R. Sundrum, Phys. Rev. Lett. 83:4690-3 (1999).

[3] R.C. Myers and M.J. Perry, Annals Phys. 172:304 (1986).

[4] S.W. Hawking and D.N. Page, Commun. Math. Phys. 87:577 (1983).

[5] D. Birmingham, Class. Quant. Grav. 16:1197-1205 (1999).

[6] A. Chamblin, S.W. Hawking, H.S. Reall, Phys. Rev. D61:065007 (2000).

[7] S. Giddings and E. Katz J. Math. Phys. 42:3082-3102 (2001); T. Banks and W. Fischler, Preprint hep-th/9906038 S. Dimopoulos and G. Landsberg, Phys. Rev. Lett. 87:161602 (2001); S.B. Giddings and S. Thomas, Phys. Rev. D65:056010 (2002); D.M. Eardley and S.B. Giddings, Phys. Rev. D66:044011 (2002) gr-qc/0201034

[8] L.A. Anchordoqui, J.L. Feng, H. Goldberg and A.D. Shapere, Phys. Rev. D65:124027 (2002) hep-ph/0112247

[9] N. Dadhich et. al. Phys. Lett. B487:1 (2000); C. Germani and R. Maartens, Phys. Rev. D64:124010 (2001); R. Casadio, et.al. Phys. Rev. D65:084040 (2002); T. Shiromizu, K. I. Maeda and M. Sasaki, Phys. Rev. D62:024012 (2000).

[10] P. Kanti and K. Tamvakis, Phys. Rev. D65:084010 (2002) hep-th/0110298 P. Kanti, I. Olasagasti and K. Tamvakis Phys. Rev. D68:124001 (2003) hep-th/0307201

[11] S.B. Giddings et.al. JHEP0003:023 (2000); J. Garriga and T. Tanaka, Phys. Rev. Lett. 84:2778 (2000).

[12] A. Chamblin et.al. Phys. Rev. D63:064015 (2001); T. Wiseman, Phys. Rev. D65:124007 (2002).

[13] R. Casadio and L. Mazzacurati, Mod. Phys. Lett. A18: 651-660 (2003) gr-qc/0205129

[14] R. Gregory and R. Laflamme, Phys. Rev. Lett. 70:2837-40 (1993); R. Gregory and R. Laflamme, in "General relativity and relativistic astrophysics" p.190-19, edited by R.B. Mann and R.G. McLenaghan, River Edge, N.J. (1993).

[15] L. Anchordoqui, H. Goldberg and A. D. Shapere, Phys. Rev. D64:024033 (2002) hep-ph/0204228

[16] M. Smolyakov and I. Volobuev, Preprint hep-th/0208025.

[17] N. Deruelle, Preprint gr-qc/0301036

[18] See also the review by V.A. Rubakov, Phys. Usp. 44:871-893 (2001) hep-ph/0104152

[19] I. Rácz and R. M. Wald, Class. Quant. Grav. 9:2643-2656 (1992).

[20] The notation: bulk indices are upper case Latin letters, brane indices are Greek letters, $G_{5}$ is Newton's constant in five dimensions, $\gamma_{\mu \nu}$ is the induced metric on the brane, and $K_{\mu \nu}$ is the extrinsic curvature of the brane.

[21] W. Israel, Nuovo Cimento B44:1 (1966); B48:463 (1966).

[22] D. P. Banerjee, Amer. J. Math 64:72-80 (1940); D. P. Banerjee, Proc. Cambridge Philos. Soc., vol. 34:30-32 (1938).

[23] W. Goldberger and M. Wise, Phys. Rev. Lett. 83:4922 (1999); T. Tanaka and X. Montes, Nucl. Phys. B582:259 (2000). 